\title{
Fatores associados à vacinação contra a influenza em idosos
}

\author{
Priscila Maria Stolses Bergamo Francisco, ${ }^{1}$ Maria Rita Donalisio, ${ }^{1}$ \\ Marilisa Berti de Azevedo Barros, ${ }^{1}$ Chester Luis Galvão César, ${ }^{2}$ \\ Luana Carandina ${ }^{3}$ e Moisés Goldbaum ${ }^{4}$
}

Como citar Francisco PMSB, Donalisio MR, Barros MBA, César CLG, Carandina L, Goldbaum M. Fatores associa-

dos à vacinação contra a influenza em idosos. Rev Panam Salud Publica. 2006;19(4):259-64.

RESUMO Objetivo. Investigar o perfil epidemiológico dos idosos que participam ou não de campanhas de vacinação contra a influenza e identificar as variáveis que poderiam influenciar a participação. Método. Estudo transversal de base populacional utilizando dados referentes à população com 60 anos ou mais residente nos municípios de São Paulo, Itapecerica da Serra, Embu, Taboão da Serra, Campinas e Botucatu no período de 2001 e 2002. Participaram do estudo 1908 idosos selecionados a partir de amostragem probabilística, estratificada e selecionada por conglomerados, em dois estágios. A análise dos dados foi exploratória, incluindo análise bivariada e regressão logística múltipla.

Resultados. Sessenta e seis por cento dos idosos aderiram à vacinação contra a gripe. Os fatores associados ao auto-relato de vacinação, após a análise ajustada, foram: idade $(O R=1,47$; IC95\% = 1,09 a 1,99), hipertensão arterial auto-relatada $(O R=1,39 ;$ IC95\% = 1,03 a 1,87) e escolaridade $(O R=0,64 ;$ IC95\% $=0,41$ a 0,98). A maior adesão à vacinação contra a gripe foi observada entre os indivíduos com idade igual ou superior a 70 anos e entre os hipertensos. Os idosos com 9 anos ou mais de estudo referiram menor adesão à vacinação.

Conclusões. Os resultados sugerem a necessidade de campanhas para facilitar o acesso por parte dos idosos e profissionais de saúde à informação acerca dos benefícios da vacinação.

Palavras-chave Influenza, saúde do idoso, suscetibilidade à doença, vacinação, Brasil.

Com o aumento na proporção de idosos na população brasileira, cresce

\footnotetext{
1 Universidade Estadual de Campinas (UNICAMP), Faculdade de Ciências Médicas, Departamento de Medicina Preventiva e Social, Campinas (SP), Brasil. Enviar correspondência para Maria Rita de Camargo Donalisio no seguinte endereço: DMPS/FCM/ UNICAMP, Caixa Postal 6111, CEP 13083-970, Campinas, SP, Brasil. Fone: +55-19-3788-8036; fax: +55-19-3788-8035; e-mail: donalisi@fcm.unicamp.br

2 Universidade de São Paulo (USP), Faculdade de Saúde Pública, Departamento de Epidemiologia, São Paulo (SP), Brasil.

3 Universidade Estadual de São Paulo (UNESP), Faculdade de Medicina de Botucatu, Departamento de Saúde Pública, Botucatu (SP), Brasil.

4 USP, Faculdade de Medicina, Departamento de Medicina Preventiva.
}

o número de indivíduos que atingem faixas etárias de risco para doenças crônicas e incapacidades. Cerca de $80 \%$ dos idosos no país apresentam alguma doença crônica (1-3). Nesse grupo, medidas preventivas e de proteção específicas devem ser priorizadas, devido à significativa e crescente demanda por serviços ambulatoriais, hospitalares e de reabilitação, conforme demonstram vários estudos (2).

Dentre as várias alterações fisiológicas associadas ao processo de envelhecimento, destacam-se aquelas referentes ao sistema imunitário dos idosos, à sua suscetibilidade e vulnerabilidade às infecções em comparação com os adultos jovens; os idosos são menos capazes de responder fisiológica e imunologicamente aos microorganismos invasores $(4,5)$. Nos últimos 20 anos, o número e a taxa de internação por infecções respiratórias agudas aumentaram de forma constante entre os idosos. A influenza e a pneumonia estão entre as principais causas de morbi-mortalidade nesse grupo etário $(6,7)$.

Considerando a crescente demanda por medidas para reduzir as complicações decorrentes dessas infecções, a 
vacinação contra a gripe surgiu como uma como estratégia nacional de impacto na redução da morbi-mortalidade por doenças respiratórias entre os idosos (8-10). As campanhas nacionais de vacinação contra a influenza iniciaram-se em 1999 em comemoração ao Ano Internacional do Idoso. No Estado de São Paulo, cerca de 84\% dos idosos receberam a vacina naquele ano. Já nos anos de 2000, 2001 e 2002, as taxas de cobertura foram de $63,9 \%$, $66,6 \%$ e $65,6 \%$, respectivamente (8). Apesar da ampla divulgação das campanhas e dos benefícios da vacinação, muitos idosos ainda não aderiram a essa prática no país. Nos Estados Unidos, estima-se que 900 mortes e 1300 hospitalizações possam ser prevenidas por cada milhão de pessoas vacinadas contra a influenza (7).

Dados do sistema de informações hospitalares do SUS (SIH/SUS) mostram que no Estado de São Paulo, no período de 1998 a 2003, ocorreram cerca de 27700 internações decorrentes de influenza e pneumonia por ano na faixa etária de 60 anos ou mais.

$\mathrm{O}$ efeito protetor da vacina contra a influenza em idosos pode variar com a capacidade imunitária do indivíduo, a imunogenicidade da vacina e a coincidência antigênica entre a vacina e as cepas circulantes na comunidade (7). Apesar de a resposta imunitária variar entre 30 e $70 \%$, os reais benefícios se referem à prevenção de pneumonia viral primária ou bacteriana secundária e de hospitalizações, e, principalmente, à redução da mortalidade entre aqueles com doenças crônicas cardiovasculares e pulmonares (11-16).

O objetivo deste trabalho foi descrever o perfil epidemiológico do idoso conforme a realização ou não de vacina contra a influenza, bem como analisar as variáveis possivelmente relacionadas ao comparecimento à vacinação.

\section{MATERIAIS E MÉTODOS}

O presente estudo transversal de base populacional utilizou dados referentes à população de 60 anos ou mais, não institucionalizada, residente em área urbana, em domicílios particula- res ou coletivos de seis municípios do Estado de São Paulo no período de 2001 e 2002.

Os dados foram obtidos a partir de um questionário multidimensional (17). Quanto ao delineamento amostral deste inquérito, os seguintes municípios foram investigados: São Paulo, Itapecerica da Serra, Embu, Taboão da Serra, Campinas e Botucatu. Essas áreas constituíram estratos independentes, subdivididos em setores censitários classificados segundo o nível de escolaridade dos chefes de família, visando aumentar a probabilidade de pessoas do estrato mais rico da população pertencerem à amostra, viabilizando as análises segundo subgrupos sociais. A pesquisa, portanto, teve como base uma amostra probabilística da população, estratificada por conglomerados e obtida em dois estágios. No primeiro, os setores censitários foram amostrados de forma sistemática e com probabilidade de inclusão proporcional ao tamanho, expresso pelo número de domicílios existentes no setor, de acordo com a contagem populacional de 1996 do Instituto Brasileiro de Geografia e Estatística (IBGE). No segundo estágio, uma amostra sistemática simples de domicílios foi sorteada em cada setor censitário.

A partir dos 1958 registros de idosos disponíveis na amostra, foram analisadas as informações sobre a realização ou não de vacina contra a gripe segundo variáveis sócio-demográficas, indicadores da condição de saúde, mobilidade física e tabagismo.

Sexo, idade, situação conjugal, escolaridade e renda mensal da família foram as variáveis sócio-demográficas incluídas neste estudo. Os indicadores da condição de saúde foram obtidos a partir da percepção do entrevistado a respeito de sua própria saúde no momento da entrevista e 1 ano antes, além do auto-relato relativo a internação hospitalar nos últimos 12 meses e a certas doenças crônicas: hipertensão, diabetes, artrite/reumatismo/ artrose, doença renal crônica, câncer (tumor maligno), doença do coração e doença crônica de pulmão (asma/ bronquite/enfisema). Os indicadores de mobilidade física referiram-se à di- ficuldade para realizar atividades vigorosas, moderadas e leves: levantar ou carregar mantimentos; subir um lance de escadas; curvar-se, ajoelhar-se ou dobrar-se; andar um quarteirão e tomar banho ou vestir-se. Todos esses indicadores foram categorizados em muita, pouca ou nenhuma dificuldade.

Os dados referentes à idade foram classificados em grupos decenais: 60 a 69 anos, 70 a 79 e 80 anos ou mais. A variável situação conjugal considerou as categorias: casado/união estável, solteiro/separado/desquitado e viúvo. Quanto à escolaridade, os dados foram agrupados em: sem escolaridade, 1 a 4 anos de estudo, 4 a 8 anos e 9 ou mais anos de estudo. A renda familiar mensal foi subdividida em: até 1 salário mínimo, entre 1 e 3 , de 3 a 5 e mais de 5 salários mínimos.

No que se refere às condições de saúde, as respostas sobre a percepção da própria saúde foram agregadas em quatro categorias: excelente/muito boa; boa; ruim/muito ruim; e ignorada, quando outra pessoa respondeu à entrevista, tendo em vista que essa percepção envolve aspectos subjetivos que não podem ser avaliados por outro informante (18). Os motivos pelos quais alguns idosos não aderiram à vacinação foram levantados a partir da pergunta "por que não recebeu vacina contra gripe?".

A análise dos dados foi conduzida utilizando-se procedimentos para a análise de inquéritos populacionais do programa Stata que permitem incorporar os pesos distintos das observações. Na presente análise, foi incluída a variável "peso da pessoa", que equivale ao inverso da fração amostral. A sumarização dos dados foi feita a partir de análise exploratória, utilizando-se porcentagens para as variáveis categóricas e média para a variável contínua.

Realizou-se o teste bivariado de associação pelo qui-quadrado $\left(\chi^{2}\right)$ e, a seguir, a análise múltipla através do modelo de regressão logística nãocondicional. Após ajuste por idade, foram selecionadas para o modelo todas as variáveis que mostraram associação com a variável dependente (vacinação contra gripe) em nível de significância de $20 \%(P<0,20)$. Utilizou-se o 
procedimento retrógrado (backward) para a elaboração do modelo múltiplo, e a variável permaneceu no modelo se $P<0,05$. A força da associação entre as variáveis independentes e a dependente foi expressa em valores estimados, brutos e ajustados, de razão de chances (odds ratio, OR), com intervalo de confiança de 95\% (IC95\%) (19).

\section{RESULTADOS}

Dos 1958 idosos entrevistados, obtiveram-se dados de 1908 (97,4\%). A perda amostral foi devida à ausência de informações sobre vacinação contra a gripe. A média de idade dos indivíduos desta amostra foi de 69,7 anos (IC95\%: 69,0 a 70,4).

Responderam à entrevista $97,7 \%$ dos 1908 dos idosos. As outras entrevistas (44 indivíduos) foram respondidas por outro informante, isto é, cuidadores, parentes ou responsáveis pelo idoso, diante da impossibilidade deste de responder. Não houve diferença no status vacinal contra a gripe comparando-se os grupos com diferentes informantes $(P=0,61)$.

A proporção de idosos que relatou vacinação contra a gripe nos 12 meses anteriores à entrevista foi de $66,1 \%$ (IC95\%: 62,2 a 69,8). Não houve diferença estatística entre os sexos quanto à realização ou não de vacina $(P=$ $0,52)$. Quanto aos idosos que não receberam a vacina, os motivos relatados foram: não considerar a vacina importante $(41,4 \%)$, crença de que a vacina provoca reação $(18,5 \%)$, ter adoecido após tomar a vacina em período anterior $(5,6 \%)$ e outros motivos $(32,6 \%)$. Cerca de $2 \%$ não souberam ou não responderam. A maioria dos que não referiram vacinação pertenceu ao grupo etário de 60 a 69 anos (61,9\%). Não houve diferença entre os sexos quanto aos motivos da não adesão à vacinação $(P=0,32)$.

Na tabela 1 está apresentada a distribuição percentual das características sócio-demográficas da amostra de idosos segundo a realização de vacina contra a gripe. Nota-se que idade, escolaridade e renda familiar mensal mostraram-se estatisticamente associa-

TABELA 1. Características sócio-demográficas em indivíduos com 60 anos ou mais conforme auto-relato de vacinação, Estado de São Paulo, Brasil, 2001 a 2002

\begin{tabular}{|c|c|c|c|}
\hline Característica & No. & Relataram vacina $(\%)$ & OR (IC95\%) \\
\hline \multicolumn{4}{|l|}{ Sexo } \\
\hline Masculino & 905 & 67,1 & 1,00 \\
\hline Feminino & 1003 & $\begin{array}{c}65,4 \\
P=0,52\end{array}$ & $0,92(0,73$ a 1,17$)$ \\
\hline \multicolumn{4}{|l|}{ Idade (anos) } \\
\hline 60 a 69 & 1061 & 62,0 & 1,00 \\
\hline 70 a 79 & 629 & 72,1 & $1,58(1,13$ a 2,22$)$ \\
\hline 80 ou mais & 218 & $\begin{array}{c}68,8 \\
P=0,02\end{array}$ & $1,35(0,86$ a 2,13$)$ \\
\hline \multicolumn{4}{|l|}{ Situação conjugal } \\
\hline Casado/união estável & 1134 & 65,4 & 1,00 \\
\hline Solteiro/separado/desquitado & 220 & 69,5 & $1,20(0,81$ a 1,80$)$ \\
\hline Viúvo & 550 & $\begin{array}{c}66,2 \\
P=0,67\end{array}$ & $1,04(0,78$ a 1,37$)$ \\
\hline \multicolumn{4}{|l|}{ Escolaridade (anos) } \\
\hline 0 & 250 & 70,5 & 1,00 \\
\hline 1 a 4 anos & 1131 & 68,4 & $0,90(0,59$ a 1,38$)$ \\
\hline 5 a 8 anos & 185 & 66,2 & $0,82(0,43$ a 1,55$)$ \\
\hline 9 anos ou mais & 334 & $\begin{array}{c}56,3 \\
P=0,05\end{array}$ & $0,54(0,31$ a 0,93$)$ \\
\hline \multicolumn{4}{|c|}{ Renda familiar mensal (salários mínimos) ${ }^{\mathrm{b}}$} \\
\hline 1 & 217 & 75,9 & 1,00 \\
\hline$>1 \mathrm{a} \leq 3$ & 404 & 64,6 & $0,56(0,33$ a 0,94$)$ \\
\hline$>3 a \leq 5$ & 347 & 70,8 & $0,80(0,49$ a 1,33$)$ \\
\hline$>5$ & 940 & $\begin{array}{c}63,2 \\
P=0,03\end{array}$ & $0,54(0,33$ a 0,90$)$ \\
\hline
\end{tabular}

a Total de pesquisados: 1908.

b Salário mínimo vigente à época da pesquisa: março a abril de $2001=\mathrm{R} \$ 151,00$ (ao redor de US\$ 69,00); maio de 2001 a março de $2002=\mathrm{R} \$ 180,00$ (ao redor de US\$ 72,00); e abril a setembro de $2002=\mathrm{R} \$ 200,00$ (ao redor de $\mathrm{R} \$ 65,00$ ).

das ao auto-relato de vacinação, considerando o nível de significância de 0,05 .

Com relação à idade, a maior cobertura foi entre indivíduos de 70 a 79 anos $(72,1 \%)$. Desagregando as faixas etárias, o grupo de 70 a 74 anos apresentou o maior percentual de adesão à vacinação $(74,8 \%)$. Entre aqueles de 75 a 79 anos, esse percentual foi de $68,9 \%$.

A tabela 2 mostra alguns dos indicadores de morbidade avaliados neste estudo. Não houve associação estatisticamente significativa entre o tabagismo e a vacinação contra a gripe $(P=$ 0,30 ). Além disso, nenhum dos indicadores de mobilidade física esteve associado à vacinação. Observa-se ainda que apenas a hipertensão arterial e o diabetes estiveram associados à vacinação na análise univariada. $\mathrm{O}$ efeito da nova variável criada (interação entre tais enfermidades) foi verificado com o teste do $\chi^{2}$, porém não apresentou significância estatística no modelo logístico múltiplo.
$\mathrm{Na}$ análise múltipla, obteve-se um modelo final com as variáveis idade, escolaridade e hipertensão arterial (tabela 3). Tanto a idade quanto a hipertensão arterial estiveram positivamente associadas à maior adesão à vacinação contra gripe. $\mathrm{O}$ auto-relato de vacinação diminuiu quando o idoso referiu 9 anos ou mais de estudo.

\section{DISCUSSÃO}

Este estudo foi realizado a partir de um inquérito domiciliar de saúde que contemplou diferentes estratos socioeconômicos em regiões heterogêneas do ponto de vista demográfico, urbano e de oferta de serviços de saúde. Dentre suas limitações, deve ser considerada a menor precisão das estimativas de proporção em função do delineamento amostral por conglomerados $(20,21)$. Além disso, os dados analisados se referem ao auto-relato de vacinação con- 
TABELA 2. Indicadores de auto-avaliação de saúde, morbidade e mobilidade física em indivíduos com 60 anos ou mais segundo vacinação contra gripe, Estado de São Paulo, Brasil, 2001 a 2002

\begin{tabular}{|c|c|c|c|c|}
\hline Indicador & $\begin{array}{l}\text { Vacinados } \\
(\%)\end{array}$ & $P$ & OR(IC95\%) & $\begin{array}{l}\text { OR ajustado } \\
(\text { IC95\%) }\end{array}$ \\
\hline \multicolumn{5}{|l|}{ Percepção da própria saúde } \\
\hline Excelente/muito boa & 66,3 & & 1,00 & 1,00 \\
\hline Boa & 66,7 & & $1,02(0,76$ a 1,36$)$ & $1,00(0,75$ a 1,33$)$ \\
\hline Ruim/muito ruim & 64,6 & 0,87 & $0,93(0,64$ a 1,33$)$ & $0,86(0,59$ a 1,25$)$ \\
\hline \multicolumn{5}{|l|}{ Saúde 1 ano antes } \\
\hline Muito melhor/um pouco melhor & 67,8 & & 1,00 & 1,00 \\
\hline Igual & 65,1 & & $0,88(0,60$ a 1,30$)$ & $0,88(0,59$ a 1,29$)$ \\
\hline Umm pouco pior/muito pior & 68,2 & 0,63 & $1,02(0,70$ a 1,48$)$ & $0,94 \quad(0,65$ a 1,38$)$ \\
\hline \multicolumn{5}{|l|}{$\begin{array}{l}\text { Internação hospitalar nos últimos } \\
12 \text { meses }\end{array}$} \\
\hline Nenhuma & 66,0 & & 1,00 & 1,00 \\
\hline Uma ou mais & 66,4 & 0,93 & $1,02(0,71$ a 1,45$)$ & $0,98(0,69$ a 1,41$)$ \\
\hline \multicolumn{5}{|l|}{ Tabagismo } \\
\hline Não & 67,4 & & 1,00 & 1,00 \\
\hline Sim & 64,5 & 0,30 & $0,88(0,69$ a 1,12$)$ & $0,90(0,71$ a 1,14$)$ \\
\hline \multicolumn{5}{|l|}{$\begin{array}{l}\text { Auto-relato de doenças/condições } \\
\text { crônicas }^{b}\end{array}$} \\
\hline Hipertensão & 70,5 & 0,01 & $1,48(1,08$ a 2,01$)$ & $1,46(1,07$ a 1,99$)$ \\
\hline Diabetes & 73,9 & 0,04 & $1,54(1,01$ a 2,35$)$ & $1,55(1,01$ a 2,36$)$ \\
\hline Artrite/reumatismo/artrose & 70,9 & 0,08 & $1,36(0,96$ a 1,91$)$ & $1,33(0,93$ a 1,88$)$ \\
\hline Doença renal & 65,7 & 0,95 & $0,98(0,54$ a 1,78$)$ & $0,99(0,55$ a 1,76$)$ \\
\hline Tumor maligno & 65,6 & 0,95 & $0,98(0,45$ a 2,14$)$ & $0,88(0,40$ a 1,95$)$ \\
\hline Doença do coração & 67,4 & 0,70 & $1,07 \quad(0,75$ a 1,52$)$ & $0,98(0,70$ a 1,42$)$ \\
\hline Asma/bronquite/enfisema & 67,4 & 0,80 & $1,06(0,65$ a 1,74$)$ & $1,05(0,64$ a 1,73$)$ \\
\hline \multicolumn{5}{|l|}{ Dificuldade para realizar atividades } \\
\hline \multicolumn{5}{|l|}{ Vigorosas } \\
\hline Não & 63,9 & & 1,00 & 1,00 \\
\hline Sim & 67,0 & 0,35 & $1,14(0,86$ a 1,53$)$ & $1,05(0,77$ a 1,42$)$ \\
\hline \multicolumn{5}{|l|}{ Moderadas } \\
\hline Não & 67,4 & & 1,00 & 1,00 \\
\hline Sim & 64,2 & 0,39 & $0,87(0,62$ a 1,21$)$ & $0,74(0,52$ a 1,04$)$ \\
\hline \multicolumn{5}{|l|}{ Leves $^{c}$} \\
\hline Levantar ou carregar mantimentos & 68,1 & 0,35 & $1,14(0,86$ a 1,52$)$ & $1,02(0,74$ a 1,40$)$ \\
\hline Subir um lance de escadas & 64,5 & 0,48 & $0,89(0,64$ a 1,23$)$ & $0,78(0,56$ a 1,09$)$ \\
\hline \multicolumn{5}{|l|}{ Curvar-se, ajoelhar-se ou } \\
\hline dobrar-se & 65,0 & 0,34 & $0,88(0,66$ a 1,15$)$ & $0,80(0,61$ a 1,04$)$ \\
\hline Andar um quarteirão & 66,4 & 0,99 & $1,00(0,70$ a 1,44$)$ & $0,88(0,60$ a 1,28$)$ \\
\hline Tomar banho ou vestir-se & 63,5 & 0,62 & $0,87(0,51$ a 1,50$)$ & $0,72(0,41$ a 1,24$)$ \\
\hline
\end{tabular}

a Ajuste por idade.

${ }^{b}$ Percentual dos que disseram sim (classe de referência: resposta negativa para doença/condição crônica).

${ }^{c}$ Percentual dos que disseram sim (classe de referência: resposta negativa).

tra a gripe e não a informações registradas em carteira de vacinação. No entanto, tais relatos têm demonstrado ser indicadores sensíveis (sensibilidade de $98 \%$ ), ainda que moderadamente específicos (especificidade de $71 \%$ ) (22). Nesse sentido, o estudo contribui para revelar o perfil do idoso que tem aderido às campanhas vacinais contra a gripe, tendo em vista a escassez de informações sobre essa temática no país.

A maioria dos idosos $(66,1 \%)$ aderiu à vacinação no período estudado, mas essa proporção esteve abaixo da meta estabelecida pelo Ministério da Saúde, que é de $70 \%$ (8). Dentre os idosos que não foram vacinados, cerca de $65 \%$ alegaram motivos que se referem à falta de esclarecimento sobre a importância da vacinação nessa faixa etária, corroborando os achados de pesquisas realizadas pela Secretaria de Estado de Saúde, que identificaram como principais motivos o medo das reações da vacina e a não preocupação com a gripe (8).

Tem sido apontada a pequena participação dos médicos no incentivo à vacinação contra a influenza, fato relevante dada a valorização que os idosos dispensam à recomendação médica
(23). Um estudo realizado na Suíça concluiu que, enquanto a opinião do paciente foi um fator determinante para a sua adesão à vacinação, a falta de recomendação médica contribuiu para a maioria das oportunidades perdidas de vacinação (24). No Reino Unido, alguns autores apontam como fatores associados à não-adesão à vacina contra a gripe a ausência de fatores mórbidos de risco, uma boa percepção da própria saúde, a falta de recomendação do médico ou enfermeiro, bem como uma visão negativa a respeito da vacina (25). $\mathrm{Na}$ Espanha, mulheres galegas de 65 anos ou mais, com autopercepção favorável do estado de saúde, também apresentaram menores taxas de vacinação contra a gripe (26). Neste estudo não houve associação entre a percepção da própria saúde e a vacinação.

Dentre as variáveis sócio-demográficas selecionadas, a idade, a renda familiar mensal e a escolaridade estiveram associadas à vacinação contra a gripe. Quanto à escolaridade, a menor taxa de adesão $(56,3 \%)$ ocorreu entre os idosos com mais de 9 anos de estudo e entre os que referiram renda familiar mensal acima de 5 salários mínimos $(63,2 \%)$. É possível supor que os idosos com maior escolaridade busquem soluções para seus problemas de saúde em serviços privados ou conveniados, os quais indicam pouco a vacinação, segundo alguns autores (23, 24). Sendo a recomendação médica um dos principais motivadores da vacinação em idosos, esse grupo de indivíduos estaria menos exposto às informações sobre a segurança e benefícios da vacina contra influenza (24).

Sabe-se que é baixa a escolaridade da população idosa, e ainda menor quanto mais velhas as coortes, pois remetem a momentos em que as chances de acesso à escola se davam de forma assimétrica por classe social e gênero (27). Neste estudo, apesar da associação entre a escolaridade e a renda familiar mensal do idoso $(P<0,001)$, apenas a escolaridade, dicotomizada em menos de 9 e 9 anos ou mais de estudo, permaneceu associada à vacinação contra a gripe no modelo final. Dentre outros componentes socioeconômicos, tanto a renda quanto o nível educacional refletem influências 
TABELA 3. Modelo logístico multivariado para vacinação contra a gripe em indivíduos com 60 anos ou mais, Estado de São Paulo, 2001 a 2002

\begin{tabular}{lccccc}
\hline \multicolumn{1}{c}{ Variáveis } & OR & Erro-padrão & $P$ & IC95\% & $\begin{array}{c}\text { Efeito } \\
\text { do desenho }\end{array}$ \\
\hline Idade $\geq 70$ anos & 1,47 & 0,22 & 0,01 & 1,09 a 1,99 & 2,25 \\
Escolaridade $\geq 9$ anos & 0,64 & 0,14 & 0,04 & 0,41 a 0,98 & 3,18 \\
Auto-relato de hipertensão arterial & 1,39 & 0,21 & 0,03 & 1,03 a 1,87 & 2,31 \\
\hline
\end{tabular}

sobre o perfil de saúde. No entanto, o nível educacional é um indicador mais estável da situação socioeconômica, já que exclui o impacto de flutuações dos recursos sobre a saúde. A sua variação é mais limitada do que a renda e a sua medida varia de acordo com o gênero, a etnia e a idade (28).

Foi observada menor referência de vacinação na faixa etária mais jovem (60 a 69 anos). A menor cobertura vacinal nesse grupo tem sido registrada desde os primeiros anos de campanha contra a influenza no Brasil e em outros países $(8,26,29,30)$. Estudos de avaliação do impacto da vacinação contra a gripe na mortalidade e morbidade hospitalar por doenças respiratórias no Estado de São Paulo verificaram uma redução significativa nas taxas de mortalidade e internação, com diferentes padrões entre as faixas etárias $(9,10)$. Entre os maiores de 80 anos, deve-se considerar a maior dificuldade de locomoção até os locais de vacinação, o que poderia justificar as menores coberturas $(68,8 \%)$.

Das condições de saúde avaliadas neste trabalho, tanto a hipertensão arterial quanto o diabetes estiveram estatisticamente associados à vacinação; no entanto, apenas a hipertensão permaneceu no modelo logístico multititucionalização de idosos em Belo Horizonte, Brasil. Rev Saúde Pública. 1999;33(5): 45460.

2. Brasil, Ministério da Saúde. Redes estaduais de atenção ao idoso. Guia operacional e portarias relacionadas. Brasília, DF: Ministério da Saúde; 2002. (Serie A: Normas Técnicas).

3. Ramos LR, Garcia JT. Terapêutica medicamentosa no idoso. Em: Prado FC, Ramos JA, Valle JR, eds. Atualização terapêutica. São Paulo: Artes Médicas; 2003. Pp. 555-8. variado. A análise dos dados foi controlada pela idade, devido a seu efeito direto no risco de morte e na ocorrência de muitas doenças. Principalmente nos grupos etários mais avançados, a idade passa a ter um efeito modificador, na medida em que altera a magnitude do risco de outros fatores (19).

Neste estudo, a prevalência de hipertensão auto-relatada foi de $51 \%$, confirmando observações realizadas por outros autores. Porém, a morbidade auto-relatada pode subestimar a prevalência de doenças ou condições crônicas, devido a problemas de memória ou ausência de diagnóstico $(18,31,32)$.

A associação entre a hipertensão e a vacinação contra a influenza permite sugerir algumas hipóteses. Por se tratar de uma doença crônica de alta prevalência, com disponibilidade de atendimento na rede pública, a hipertensão arterial traz o indivíduo ao serviço de saúde com maior freqüência para controle dos níveis pressóricos, busca de medicação e exames periódicos. Nesse sentido, o paciente hipertenso pode ter maior acesso às informações sobre campanhas vacinais, maior contato com a equipe de saúde e outros tipos de divulgação. Habituado a freqüentar a unidade básica de saúde, comparece com maior freqüência às campanhas.

\section{REFERÊNCIAS}

4. Fabra AR. Neumonías en el anciano. Antibiot Infecc. 1996;4(2):5-13.

5. Dodet B. Immunity in the elderly. Vaccine. 2000;18(16):1565.

6. Cruz AM, Bravo J, Rojas V. Conocimientos, creencias y prácticas respecto a las infecciones respiratorias agudas en adultos mayores de 65 años. Cad Saude Publica. 1999;15(4): 851-7.

7. Centers for Disease Control and Prevention (CDC). Prevention and Control of InfluenzaRecommendations of the Advisory Commit-
Portadores de outras doenças crônicas menos prevalentes na população deveriam, da mesma forma, estar expostos à divulgação sobre a vacina, porém nem sempre o serviço público está equipado para seguimento e tratamento dessas doenças, de forma que o doente é encaminhado para outros serviços. Além disso, médicos das diversas especialidades clínicas têm deixado de indicar a vacinação aos seus pacientes em serviços públicos e privados (8).

A associação entre a vacinação e $o$ diabetes apenas na análise univariada, bem como a ausência de associação entre outras doenças crônicas e a imunização, sugerem que esses indivíduos de risco não estão sendo priorizados. Vale ressaltar a indicação formal da vacinação nesses pacientes devido à maior gravidade das complicações clínicas decorrentes da infecção pelo vírus da influenza $(7,33)$.

A infecção pelo vírus da influenza pode ser considerada benigna, autolimitada e prevenível; porém, em grupos de risco, passa a ter maior importância epidemiológica devido às suas complicações $(2,7,34)$. Há que intensificar o repasse de informações sobre a vacinação do idoso nos serviços públicos e privados, para todas as faixas etárias e, particularmente, para os portadores de doenças crônicas, no sentido de estender as coberturas vacinais e ampliar os seus benefícios. Apesar do aumento nas taxas de cobertura nos anos de 2003 e 2004 (74,6 e 78,06\%, respectivamente), o número de idosos a serem vacinados ainda é expressivo (8). São necessárias campanhas específicas endereçadas aos grupos de maior risco e aos menos vacinados. tee on Immunization Practices (ACIP). MMWR. 2005;54(08):1-40.

8. Estado de São Paulo, Coordenação dos Institutos de Pesquisa, Centro de Vigilância Epidemiológica. Campanha de vacinação para o idoso-2005. São Paulo: SES; 2005. (Informe Técnico).

9. Francisco PMSB, Donalisio MRC, Latorre MRO. Internações por doenças respiratórias em idosos e a intervenção vacinal contra influenza no Estado de São Paulo. Rev Bras Epidemiol. 2004;7(2):220-7. 
10. Francisco PMSB, Donalisio MRC, Latorre MRO. Vacinação contra influenza e mortalidade por doenças respiratórias em idosos, 1980-2000. Rev Saude Publica. 2005;39(1):75-81.

11. Fedson DS, Wajda A, Nicol JP, Hammond GW, Kaiser DL, Roos LL. Clinical effectiveness of influenza vaccination in Manitoba. JAMA. 1993;270(16):1956-61.

12. Ahmed AE, Nicholson KG, Nguyen-Van-Tam JS. Reduction in mortality associated with influenza vaccine during 1989-90 epidemic. Lancet. 1995;346(8975):591-5.

13. Gross PA, Hermogenes AW, Sacks HS, Lau J, Levandowski RA. The efficacy of influenza vaccine in elderly persons. A meta-analysis and review if the literature. Ann Intern Med. 1995;123(7):518-27.

14. Nichol KL, Margolis KL, Wuorenma J, Von Sternberg T. The efficacy and cost effectiveness of vaccination against influenza among elderly persons in the community. $\mathrm{N}$ Engl J Med. 1994;331(12):778-84.

15. Nichol KL, Wuorenma J, Von Sternberg T. Benefits of influenza vaccination for low-, intermediate-, and high-risk senior citizens. Arch Intern Med. 1998;158(16):1769-76.

16. Bridges CB, Winquist AG, Fukuda K, Cox NJ, Singleton JA, Stikas RA, et al. Prevention and control of influenza: Recommendations of the Advisory Committee on Immunization Practices (ACIP). MMWR Recomm Rep. 2003;52 (RR-8):1-34; quiz CE1-4.

17. Cesar CLG, Carandina L, Alves MCGP, Barros MBA, Goldbaum M. Saúde e condições de vida em São Paulo-inquérito multicêntrico de saúde no Estado de São Paulo (ISA-SP). São Paulo: USP/FSP; 2005.

18. Lima-Costa MF, Barreto MS, Giatti L. Condições de saúde, capacidade funcional, uso de serviços de saúde e gastos com medicamentos da população idosa brasileira: um estudo descritivo baseado na Pesquisa Nacional por Amostra de Domicílios. Cad Saude Publica. 2003;19(3):735-43

19. Hosmer DW, Lemeshow S. Applied logistic regression. New York: John Wiley \& Sons; 1989.

20. Cochran WG. Sampling techniques. New York: John Wiley \& Sons; 1977.

21. Korn EL, Graubard BI. Epidemiologic studies utilizing surveys: accounting for sampling design. Am J Public Health. 1991;81(9):1166-73.

22. Mac Donald R, Baken L, Nelson A, Nichol KL. Validation of self-report of influenza and pneumococcal vaccination status in elderly outpatients. Am J Prev Med. 1999:16(3):173-7.

23. Secretaria de Estado de Saúde de São Paulo, Centro de Referência em Saúde do Trabalhador de São Paulo. Pesquisas indicam pequena participação dos médicos no incentivo à vacinação contra influenza. Rev Saude Publica. 2004;38(4):607-8.

24. Bovier PA, Chamot E, Gallacchi MB, Loutan $\mathrm{L}$. Importance of patients' perceptions and general practitioners' recommendations in understanding missed opportunities for immunisations in Swiss adults. Vaccine. 2001; 19(32):4760-7.

25. Evans MR, Watson PA. Why do older people not get immunised against influenza? A community survey. Vaccine. 2003;21(19-20): 2421-7.

26. Pena-Rey I, Perez-Farinos N, Sarria-Santamera A. Factors associated with influenza vaccination among elderly Spanish women. Public Health. 2004;118(8):582-7.

27. Berquó E, Baeninger R. Os idosos no Brasil: considerações demográficas. Textos Nepo [site da Internet]. Disponível em: http://www. nepo.unicamp.br/textos_publish/textos nepo_37.pdf. Acessado em 3 abril de 2006.

28. Becket M. Converging health inequalities in later life-an artifact of mortality selection? J Health Soc Behav. 2000;41(1):106-19.

29. Pregliasco F, Sodano L, Mensi C, Selvaggi MT, Adamo B, D'Argenio P, et al. Influenza vaccination among the elderly in Italy. Bull World Health Organ. 1999;77(2):127-31.

30. Dannetun E, Tegnell A, Normann B, Garpenholt $\mathrm{O}$, Giesecke J. Influenza vaccine coverage and reasons for non vaccination in a sample of people above 65 years of age, in Sweden, 1998-2000. Scand J Infect Dis. 2003;35(6-7): 389-93.

31. Piccini RX, Victora CG. Hipertensão arterial sistêmica em área urbana no sul do Brasil: prevalência e fatores de risco. Rev Saude Publica. 1994;28(4):261-7.

32. Firmo JOA, Lima-Costa MF, Uchôa E. Projeto Bambuí: maneiras de pensar e agir de idosos hipertensos. Cad Saude Publica. 2004;20(4): 1029-40.

33. Rocha JLL, Baggio HCC, Cunha CA, Niclewicz EA, Leite SAO, Baptista MIDK. Aspectos relevantes da interface entre diabetes mellitus e infecção. Arq Bras Endocrinol Metab. 2002; 46(3):221-9.

34. Govaert TM, Thijs CT, Masurel N, Sprenger MJ, Dinant GJ, Knottnerus JA. The efficacy of influenza vaccination in elderly individualsA randomized double-blind placebo-controlled trial. JAMA. 1994;272(21):1661-5.

Manuscrito recebido em 18 abril de 2005. Aceito em versão revisada em 27 de janeiro de 2006.

ABSTRACT Objective. To investigate the epidemiologic profile of elderly persons who do or do not participate in influenza vaccination campaigns and to identify the variables that bear an influence on participation.

Factors associated with vaccination against influenza in the elderly

Method. A cross-sectional population-based study was performed using data on individuals aged 60 years or older who were living in the municipalities of São Paulo, Itapecerica da Serra, Embu, Taboão da Serra, Campinas and Botucatu, Brazil, in 2001 and 2002. A stratified random sample of 1908 elderly individuals was selected by means of two-stage cluster sampling. Exploratory data analysis was performed, including bivariate analysis and multiple logistic regression.

Results. Sixty-six percent of the elderly subjects reported having received vaccination against influenza. After adjustment, the following factors were found to be associated with having received vaccination, based on self-report: age (OR $=1.47 ; 95 \%$ $\mathrm{CI}=1.09$ to 1.99$)$, self-reported hypertension $(\mathrm{OR}=1.39 ; 95 \% \mathrm{CI}=1.03$ to 1.87$)$ and educational level ( $\mathrm{OR}=0.64 ; 95 \% \mathrm{CI}=0.41$ to 0.98$)$. The highest number of vaccinated individuals was observed in the group $\geq 70$ years of age and in the hypertension group. Individuals with 9 or more years of schooling reported less adherence to influenza vaccination.

Conclusions. The results suggest the need for campaigns to make information on the benefits of influenza vaccination more easily accessible to the elderly and health professionals.

Key words Influenza, aging health, disease susceptibility, vaccination, Brazil. 